\title{
Formation of SiO and Related Si-Based Materials Through Carbothermic Reduction of Silica-Containing Slag
}

\author{
S. V. Komarov ${ }^{1}$, D. V. Kuznetsov ${ }^{2}$, V. V. Levina ${ }^{2}$ and M. Hirasawa ${ }^{1}$ \\ ${ }^{1}$ Institute of Multidisciplinary Research for Advanced Materials, Tohoku University, Sendai 980-8577, Japan \\ ${ }^{2}$ Moscow Steel and Alloys Institute (Technical University), Leninsky prospect 4, Moscow 117417, Russia
}

\begin{abstract}
Silicon based nanoparticulates of various composition and morphology have been produced by smelting reduction method which includes a carbothermic reduction of $\mathrm{SiO}_{2}-\mathrm{Al}_{2} \mathrm{O}_{3}-\mathrm{CaO}$ starting materials to $\mathrm{SiO}$ vapor at $2073 \mathrm{~K}$ and transfer of the vapor with a carrier-gas to cooler surfaces inside the experimental reactor where the nanoparticulates were deposited. The chemical composition of the starting materials was matched to the basic composition of silica-rich coal ash which is considered as a potential source of Si in this study. Special emphasis was placed upon examining the degree of $\mathrm{SiO}_{2}$ reduction from starting materials, purity and morphology of the as-obtained nanomaterials. It is shown that up to $20 \%$ of Si can be converted from the silica-based melt into rounded nanoparticles, nanoparticle chains and nanowires containing Si,O and $\mathrm{C}$ in variable proportions depending on deposition temperature and gas flow conditions. The diameter of nanoparticulates was estimated to be in the range of $20 \sim 100 \mathrm{~nm}$. The nanoparticles and chains were found to be deposited at lower temperature locations (293 1320 K) while the nanowires were obtained at higher temperatures $(1320 \sim 1570 \mathrm{~K})$. There was a tendency for an increase in Si concentration in order of nanowires, nanoparticles chains and nanoparticles. The carbon concentration, on the contrary, was much higher in nanowires as compared to that in nanoparticles. Although the degree of $\mathrm{SiO}_{2}$ reduction from silica-containing melt to $\mathrm{SiO}$ vapor is limited because a part of $\mathrm{SiO}{ }_{2}$ reacts with $\mathrm{C}$ producing $\mathrm{SiC}$, its good controllability, high productivity and possibility for processing cheap starting materials make the smelting reduction a very attractive technique for production of silicon based nanostructured materials.
\end{abstract}

(Received September 24, 2004; Accepted February 7, 2005; Published April 15, 2005)

Keywords: silicon-based nanoparticulate, silica-containing melt, carbothermic reduction process, degree of $\mathrm{SiO}_{2}$ reduction, product purity, process chemistry

\section{Introduction}

Owing to their unique properties, silicon and its compounds continue to play an important role in designing semiconductor and optoelectronic devices. Over the last years, increasing demands for miniaturization of electronic systems have become a key driver of developing advanced micro- and nanostructured Si-based materials as well as methods for their production. Recently, several routes for synthesis of Si-based nanowires, nanospheres, nanoagglomerates have been experimentally verified including thermal evaporation and laser ablation. The synthesis principle is based on the ability of $\mathrm{Si}$ to form a volatile monoxide $\mathrm{SiO}$ in the presence of oxygen in the system. In the experiments, commercially available $\mathrm{SiO},{ }^{1,2)} \mathrm{SiO}_{2}{ }^{3)}$ or a $\mathrm{Si}-\mathrm{SiO}_{2}$ mixture $^{4,5)}$ was heated up in an inert gas atmosphere under a lower pressure. This resulted in formation of $\mathrm{SiO}$ vapor and its following condensation on cooler parts of the experimental reactors where the $\mathrm{SiO}$ vapor was transferred by the inert carrier gas. The experiments showed that temperature is the process key parameter in controlling both the $\mathrm{SiO}$ evaporation rate and morphology of nanostructured particles condensed. Although the temperature of heating sample was varied depending on material system used, $1600^{\circ} \mathrm{C}$ for $\mathrm{SiO}_{2}, 1400^{\circ} \mathrm{C}$ for $\mathrm{Si}-\mathrm{SiO}_{2}$ mixture and $1100^{\circ} \mathrm{C}$ for $\mathrm{SiO}$, the tendency for increasing the yield of nanostructured product with temperature was similar for all these studies. Besides, the yield of $\mathrm{Si}$ nanowires was found to increase with increasing pressure of Ar carrier gas. ${ }^{1)}$ However, because the working temperatures of the above methods are comparatively low, they are very limited in quantity of synthesized Sibased nanomaterials.

Constantly increasing demands for the nanometersized Sibased materials will cause the need for production of the $\mathrm{Si}$ - based nanomaterials in a larger amount. The above findings suggest that one of the processes, which could meet this need is smelting reduction. This process is one of the commonly used techniques in pyrometallurgy for reductive refining of oxide-based materials. The process involves supplying energy to melt starting materials in an appropriate reactor and performing relevant chemical reactions in the molten bath under controlled conditions such as gas atmosphere, temperature and pressure inside the reactor. Because the process is performed at very high temperatures, it offers better productivity as compared with other processes.

The concept of such a process has been reported by one of the authors of the present study. ${ }^{6}$ Although the proposed two-stage reduction process was aimed at producing silicon, it has been shown that a vigorous formation of $\mathrm{SiO}$ vapor can be achieved by reducing pure molten silica by solid silicon carbide at a temperature of $2073 \mathrm{~K}$ and higher under a lower pressure in the system. Based on the proposed concept, we undertook the present study to examine experimentally the applicability of the smelting reduction processes for synthesis of micro- and nanostructured silicon based materials. The synthesis principle is based on the following three steps: carbothermic reduction of silica-containing slag to $\mathrm{SiO}$ vapor, transport of the vapor by a carrier gar, and condensation of $\mathrm{SiO}$ from the vapor at cooler parts of reaction chamber. Depending on temperature and gas phase composition, after condensation $\mathrm{SiO}$ can undergo further chemical reactions producing silicon, silicon suboxide or silicon carbide. A premelted slag composed of $\mathrm{SiO}_{2}, \mathrm{Al}_{2} \mathrm{O}_{3}$ and $\mathrm{CaO}$ in a molar ratio of $1.0 / 0.17 / 0.15$, was used as a starting material. This ratio corresponds to that of the basic components in silica-rich coal ash which is considered as a potentially usable source of $\mathrm{Si}$ in the present study. Such a coal ash is generated in huge quantity as a by-product during 
combustion of some kinds of higher-rank coals.

In the present study, we examined effects of gas flow and temperature inside the reaction chamber on the degree of $\mathrm{SiO}_{2}$ reduction from silica-containing melt, on purity and morphology of the as-obtained product. For a better understanding of the mechanism of the chemical reactions involved in the process, composition of exhaust gas and slag residue were also investigated. Chemical analysis was performed by an electron probe microanalyser (EPMA) with an energy and wave dispersive X-ray spectrometers. Phase composition of slag residue was investigated by X-Ray diffraction (XRD) method. Size and shape of the obtained nanomaterials were observed with a scanning electron microscope (SEM). More comprehensively, characteristics of the synthesized nanomaterials will be discussed in our next paper.

\section{Experimental Setup and Procedure}

Figure 1 is a schematical representation of experimental apparatus. A crucible (I.D. $0.04 \mathrm{~m}$, height $0.17 \mathrm{~m}$ ), made of graphite (density $1850 \mathrm{~kg} \cdot \mathrm{m}^{-3}$, total impurity $<500 \mathrm{ppm}$ ) was put inside a sealed water-cooled reaction chamber made of quartz. The chamber was installed coaxially with an induction coil powered by a $15 \mathrm{~kW}, 100 \mathrm{kHz}$ generator. The experiments were performed under an atmosphere of highly purified Ar gas introduced into the chamber through an inlet tube at a certain flow rate under a pressure close to atmospheric one. The graphite crucible was covered by a graphite threaded lid with three holes, one of which was drilled at the lid center and the others were close to the lid edge. The latter two holes served to let Ar gas flow into the graphite crucible from the inner space of the chamber. Ar gas flew over the crucible and then entered an outlet graphite tube (I.D. $0.01 \mathrm{~m}$ ) fixed at the lid center hole so that the lower end of tube was separated from the crucible bottom by a certain distance, $h$. The upper end of the tube was jointed with an outlet alumina tube of the same diameters which, in turn, was connected to a holder of a glass fiber filter. In some experiments a different type of the graphite tube was used. The tube consisted of a graphite outer tube (I.D. $12 \mathrm{~mm}$ ) and 12 graphite rings (I.D. and height $10 \mathrm{~mm}$ ) enclosed in the outer tube (not shown here).

During an experiment run, exhaust gas, leaving the

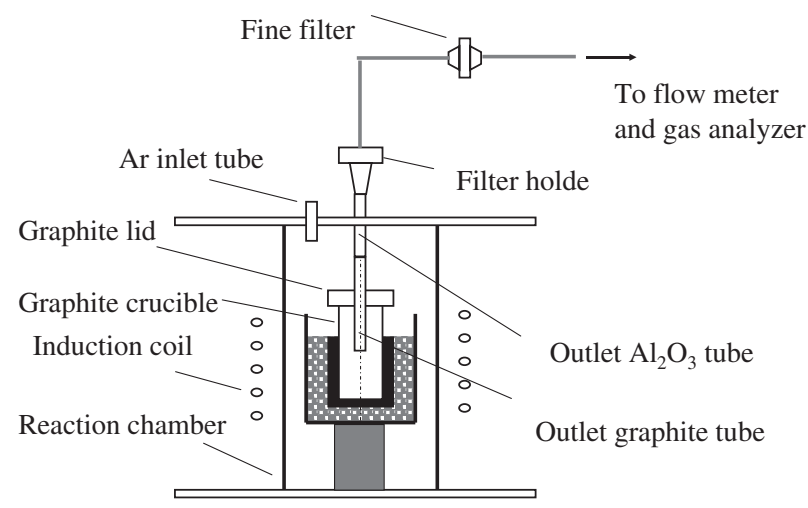

Fig. 1 Experimental setup. graphite crucible, was analyzed for $\mathrm{CO}$ and $\mathrm{CO}_{2}$ continuously by an IR-analyzer after passing through a fine filter. The flow rate of the exhaust gas was measured by means of a wet flow meter. All the measurement data were stored by a PC.

The slag, or model coal ash, was prepared as follows. Predetermined amounts of reagent grade of $\alpha-\mathrm{Al}_{2} \mathrm{O}_{3}, \mathrm{SiO}_{2}$ (quartz) and $\mathrm{CaO}$ (calcined from $\mathrm{CaCO}_{3}$ ) were mixed and melted in the induction furnace at a temperature of about $1873 \mathrm{~K}$. The slag was fully glassy and no segregation nor deposited phase was observed. The slag contained $\mathrm{SiO}_{2}$ (68.64 mass\%), $\mathrm{Al}_{2} \mathrm{O}_{3}$ (22.62 mass\%) and $\mathrm{CaO}$ (8.75 mass\%) as verified by EPMA analysis. The slag was crushed and mixed with a pure powdered carbon in a molar ratio of 0.7 relative to amount of $\mathrm{SiO}_{2}$ in the slag. This ratio was chosen from assumption that the remainder of carbon should come from the graphite crucible. Then, the slag-carbon mixture was pelletized with a 8 -mm diameter die. A very small amount of aqueous starch solution was added as a binder. The pellets were dried at $105^{\circ} \mathrm{C}$ for $24 \mathrm{~h}$ to remove the remaining moisture.

The experimental procedure was as follows. The graphite crucible was heated to the desired temperature under an atmosphere of $\mathrm{Ar}$ of the constant flow rate ranged within $2.5 \sim 33.3 \times 10^{-6} \mathrm{Nm}^{3} \cdot \mathrm{s}^{-1}$. The temperature was measured by a W/Re- thermocouple (W-5\%Re vs. W-26\%Re), which was inset inside the graphite crucible through the graphite and alumina tubes from the top. The tip of the thermocouple was placed at a distance of $0.01 \mathrm{~m}$ above the crucible bottom. In the present study, all experiments were made at $2073 \mathrm{~K}$. After the temperature reached this temperature, a few pellets (total mass about 5 $\sim \mathrm{g}$ ) were loaded into the crucible. This moment was considered as starting time of the experiment. Each experiment was continued typically for $30 \mathrm{~min}$. After experiment, the reaction chamber was cooled down to room temperature under Ar atmosphere.

In the present study, particular emphasis has been placed on the chemical composition of the as-obtained product. The concentration of $\mathrm{Si}, \mathrm{Al}, \mathrm{Ca}, \mathrm{C}$ and $\mathrm{O}$ was measured by means of EPMA (JEL JXA-8200) equipped with the energy dispersive $\mathrm{X}$-ray (EDX) and wave length dispersive X-ray (WDX) analysis. The same techniques were used also to investigate the element distribution over crucible residues. The measurements were performed at a beam accelerating voltage of $15 \mathrm{kV}$ and current of $30 \mathrm{nA}$ under a take-off angle of $38^{\circ}$.

XRD technique (Rigaku, RINT 2200) was used to detect the crystalline phases presented in the crucible residues. The $\mathrm{XRD}$ patterns were taken with $\mathrm{Cu} K \alpha$ radiation over the range of 10 to $80^{\circ}$ at a speed of $0.05^{\circ} \mathrm{s}^{-1}$. The crucible residues were also examined by a digital microscope for the observation of the constitution of the residues after experiment. For this purpose, sections were cut out from residue containing crucible parts and were polished with $1 \mu \mathrm{m}$ diamond powder.

A scanning electronic microscope (SEM, Hitachi, S4100L) was applied to examine the particle size and surface morphology of the materials yielded in the experiments. The SEM micrographs were taken at an accelerating voltage of $15 \mathrm{kV}$ and working distance of $5 \sim 15 \mathrm{~mm}$. 


\section{Experimental Results and Discussion}

\subsection{Chemical reactions in the melt}

A number of chemical reactions can result in $\mathrm{SiO}$ formation under the given experimental conditions. Initially, the molten slag components $\left(\mathrm{SiO}_{2}, \mathrm{Al}_{2} \mathrm{O}_{3}\right.$ and $\left.\mathrm{CaO}\right)$ can react with carbon at the surfaces of carbon particles or graphite crucible wall. These reactions can be termed as primary. To make an estimate of what species would be formed as a results of these reactions, partial pressures of gaseous matters, amounts of liquid and solid matters (if exist) were calculated for corresponding equilibrium states under experimental temperature of $2073 \mathrm{~K}$ and pressure of $0.1 \mathrm{MPa}$ by using the thermochemical software and database FactSage with the built-in option of predicting component activities in glassy slag. According to the results, the dominant components of gas phase are $\mathrm{CO}$ and $\mathrm{SiO}$. Although the partial pressures of $\mathrm{SiO}, P_{\mathrm{SiO}}$, and $\mathrm{CO}, P_{\mathrm{CO}}$, were changed with molar ratio of $\mathrm{SiO}_{2}$ in slag to $\mathrm{C}$ in graphite, their sum, $P_{\mathrm{CO}}+$ $P_{\mathrm{SiO}}$ was 0.9999 regardless of the ratio.. Another feature of the reacting system is a strong trend towards formation of solid silicon carbide, SiC. Therefore, it is concluded that the system should include gaseous $\mathrm{CO}$ and $\mathrm{SiO}$, newly formed solid $\mathrm{SiC}$ and the rest of slag.

Thus, based on the above equilibrium concept, one can write the following primary reactions

$$
\begin{aligned}
& \mathrm{SiO}_{2}(\mathrm{l})+\mathrm{C}(\mathrm{s})=\mathrm{SiO}(\mathrm{g})+\mathrm{CO}(\mathrm{g}) \\
& \mathrm{SiO}_{2}(\mathrm{l})+3 \mathrm{C}(\mathrm{s})=\mathrm{SiC}(\mathrm{s})+2 \mathrm{CO}(\mathrm{g})
\end{aligned}
$$

Then, the reaction products can be involved in other reactions with slag components or carbon which can be termed as secondary. Analysis of the available literature (for example ${ }^{7)}$ ) showed that at least two secondary reactions are of prime importance for the following discussion

$$
\begin{aligned}
& 2 \mathrm{SiO}_{2}(\mathrm{l})+\mathrm{SiC}(\mathrm{s})=3 \mathrm{SiO}(\mathrm{g})+\mathrm{CO}(\mathrm{g}) \\
& \mathrm{SiO}(\mathrm{g})+2 \mathrm{C}(\mathrm{s})=\mathrm{SiC}(\mathrm{s})+\mathrm{CO}(\mathrm{g})
\end{aligned}
$$

Since reactions (1) and (3) produce $\mathrm{SiO}$, their proceeding is favorable for the transfer of Si from slag to gas phase. On the other hand, formation of $\mathrm{SiC}$ according to the reactions (2) and (4) is unfavored for the process because the $\mathrm{SiC}$ formation occurs with consumption of $\mathrm{SiO}_{2}$ or $\mathrm{SiO}$.

\subsection{Location of silicon suboxide deposition}

It was observed in the experiments that the slag- $\mathrm{C}$ pellets were melted down for a few seconds after loading. Immediately afterwards, as a result of reactions between the melt and carbon, a vigorous evolution of gaseous $\mathrm{CO}$ and $\mathrm{SiO}$ started. The gas mixture was diluted with Ar carrier gas, which flowed over the crucible. Then, Ar-CO-SiO gas was transferred into the graphite and alumina tubes. As the gas mixture passed inside the tubes, $\mathrm{SiO}$ was condensed and deposited as silicon suboxide, $\mathrm{SiO}_{x}$ on the surfaces of cooler parts inside the tubes while $\mathrm{Ar}-\mathrm{CO}$ mixture arrived at the gas analyzer and wet gas meter.

The deposit was collected at three locations: 1 - the inner wall of crucible and the outer surfaces of graphite tube, 2 the inner surfaces of graphite and alumina tubes, and 3-the filter. The deposit amount was dependent on the gas flow conditions. In the present experiments, the gas flow con-
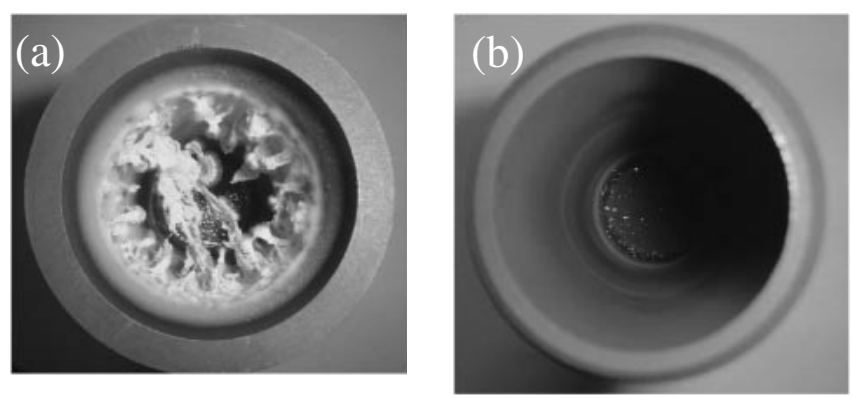

Fig. 2 Appearance of deposit on crucible wall under different flow conditions of carrier gas.

ditions were controlled by two experimental variables: the flow rate of Ar carrier gas, $V_{\mathrm{cg}}$, and the distance between graphite tube tip and crucible bottom, $h$. At larger $h$ or/and smaller $V_{\text {cg }}$, the deposition occurred mostly on the wall inside the crucible and outer surface of graphite tube. These conditions are undesirable for the process because they make the deposit growth hardly controllable. On the other hand, higher $V_{\text {cg }}$ and smaller $h$ were more favorable for collecting the deposit on the inner surfaces of graphite and alumina tubes as well as on the filter due to a fast carrying of $\mathrm{SiO}$ vapor from high temperature zone. Under these conditions, the first portion of brown-colored deposit was already observed on the filter within a minute after the pellets were loaded. However, the largest amount of deposit was collected inside the tubes. The distance $h$ appears to be more important parameter for the control of deposition location than $V_{\mathrm{cg}}$. When $h$ exceeded $0.08 \sim 0.1 \mathrm{~m}$, a significant amount of deposit was collected on the crucible wall even at higher flow rates of Ar carrier gas.

Figure 2(a,b) shows appearance of deposit on crucible wall uder different flow conditions of carrier gas: $V_{\mathrm{cg}}=$ $3.11 \times 10^{-5} \mathrm{Nm}^{3} \cdot \mathrm{s}^{-1}$ and $h=0.185 \mathrm{~m}(\mathrm{a})$, and $V_{\mathrm{cg}}=1.21 \times$ $10^{-5} \mathrm{Nm}^{3} \cdot \mathrm{s}^{-1}$ and $h=0.04 \mathrm{~m}$ (b). In Fig. 2(a), the crucible inner wall is copiously covered by a white deposit growing from the wall surface to the crucible center. This resulted in a formation of fibre-shaped fragments inside the crucible as shown in the figure. However, when $h$ was as smaller as $0.04 \mathrm{~m}$, as seen in Fig. 2(b), there is no deposit on the surface of crucible.

\subsection{The degree of $\mathrm{SiO}_{2}$ reduction and product purity}

Figure 3 shows relative mass of deposit inside the tubes and on filter, $M_{\mathrm{d}}$, as a function of flow rate of carrier gas, $V_{\mathrm{cg}}$, at a constant $h=0.04 \sim 0.045 \mathrm{~m}$. The value $M_{\mathrm{d}}$ was defined as the ratio of the mass increment of tubes during experiment to the mass of $\mathrm{SiO}_{2}$ in loaded pellets. As seen in the figure, higher gas flow rate gives larger $M_{\mathrm{d}}$, that results from the increase in mass transfer rate of $\mathrm{SiO}$ from the melt surface. A visual examination of the tubes after experiments revealed that their inner walls were completely covered with the differently colored deposit except some parts very close to the graphite tube lower tip. The color, although not shown here, was varied from light gray at lower places inside the graphite tube to brown at upper places inside the graphite and alumina tubes, and at the filter. Especially large amount of deposit was obtained at the places distanced from the lower 


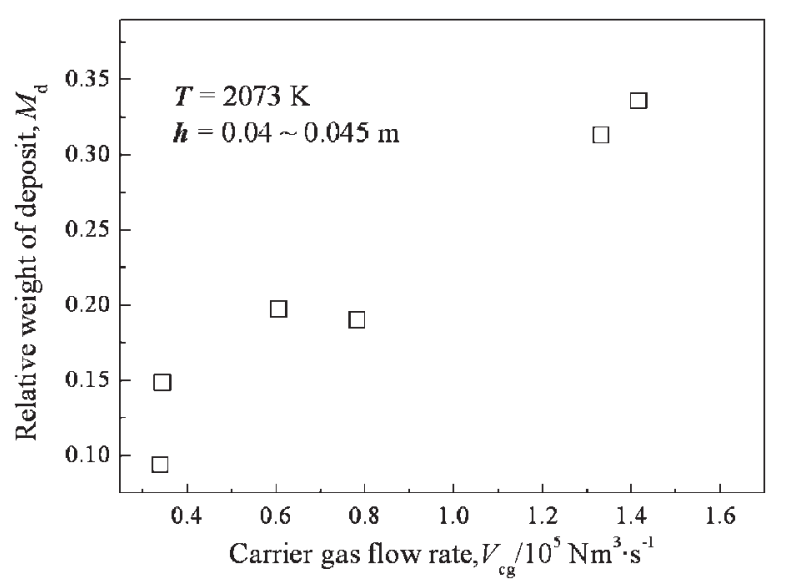

Fig. 3 Effect of carrier gas flow rate on relative weight of deposit.
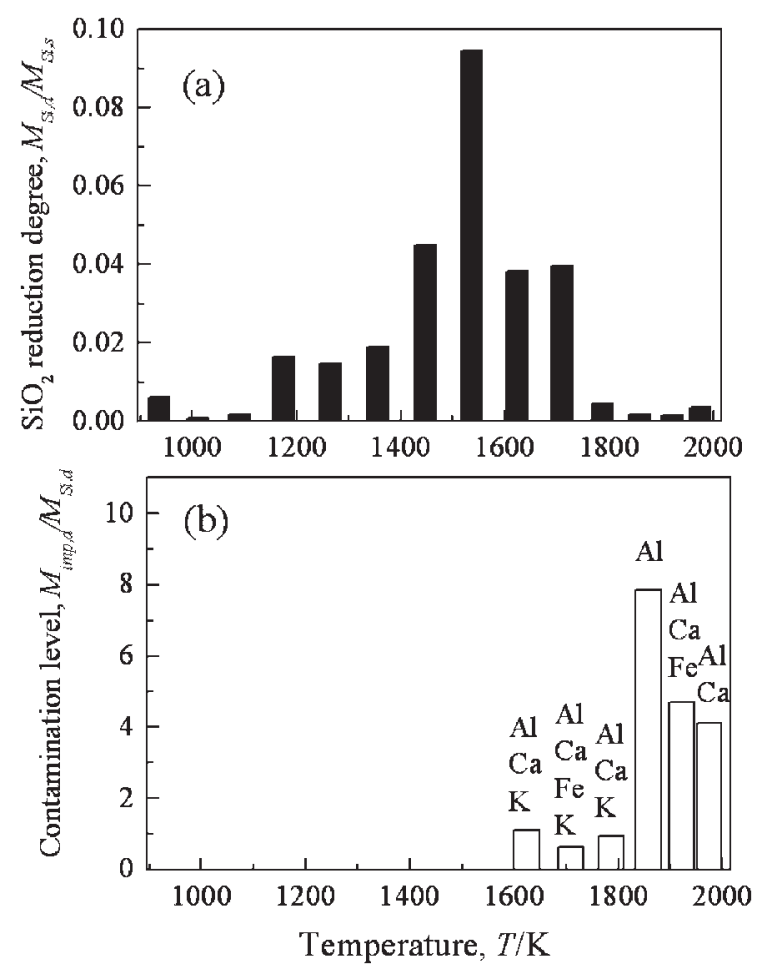

Fig. 4 Variation of $\mathrm{SiO}_{2}$ reduction degree (a) and contamination level (b) with temperature.

tip of graphite tube by $0.04 \sim 0.06 \mathrm{~m}$. This led to clogging of the tube with the deposit during experiments.

In a few experiments several graphite rings were placed inside graphite tube. The deposit mass was determined at each ring wall from the mass changes of each rings after experiments. The composition of deposit was measured by EPMA also at each ring position. Figure 4(a) presents a typical variation of the degree of $\mathrm{SiO}_{2}$ reduction from slag, $M_{\mathrm{Si}, \mathrm{d}} / M_{\mathrm{Si}, \mathrm{s}}$, with temperature inside the graphite tube. Here, $M_{\mathrm{Si}, \mathrm{d}}$ and $M_{\mathrm{Si}, \mathrm{s}}$ are the masses of Si in deposit collected by an appropriate ring and that in starting sample, respectively. The results were obtained under $V_{\mathrm{cg}}=1.77 \times 10^{-5} \mathrm{Nm}^{3} \cdot \mathrm{s}^{-1}$ and $h=0.04 \mathrm{~m}$. In the figure, the highest temperature corresponds to the tube lower tip while the lowest one pertains to an upper location inside the tube distanced from its lower tip by about $0.13 \mathrm{~m}$. The temperature inside the tube was determined from prior measurements.

As seen from Fig. 4(a), there is a temperature zone between $1440 \sim 1700 \mathrm{~K}$ within which the most amount of deposit was collected. In this zone about $20 \%$ of total deposit condensed. Concentration of metal impurities, such as $\mathrm{Al}$ and $\mathrm{Ca}$, in the deposit was strongly dependent on local temperature inside the graphite tube. Figure 4(b) shows contamination level of the as-obtained deposit with temperature inside the graphite tube. The data were obtained under the same experimental conditions as those in Fig. 4(a). The contamination level is defined as the ratio between total mass of impurities, $M_{\mathrm{imp}, \mathrm{d}}$, and $\mathrm{Si}, M_{\mathrm{Si}, \mathrm{d}}$, in the deposit. As seen in the figure, the deposit is contaminated with metal impurities at temperatures higher than $1600 \mathrm{~K}$. The metal impurities are indicated at the bars in order of decreasing their concentration from the top. The main impurities are $\mathrm{Al}$ and $\mathrm{Ca}$ entering the deposit possibly through the gas phase as relatively volatile aluminum suboxide, $\mathrm{Al}_{2} \mathrm{O}$ and calcium vapor. Thermodynamic estimations showed that, in the systems $\mathrm{Al}_{2} \mathrm{O}_{3}-\mathrm{C}$ and $\mathrm{CaO}-\mathrm{C}$, the vapor pressures of $\mathrm{Al}_{2} \mathrm{O}$ and $\mathrm{Ca}$ are much higher than those of other species containing $\mathrm{Al}$ and $\mathrm{Ca}$, respectively. For example, on the basis of thermodynamic data, at $T=2073 \mathrm{~K}$ and total pressure equals to unity, partial pressures of $\mathrm{Al}_{2} \mathrm{O}$ and $\mathrm{Ca}$, $P_{\mathrm{Al}_{2} \mathrm{O}}$ and $P_{\mathrm{Ca}}$, are calculated as $9.8 \times 10^{-3}$ and $6.5 \times 10^{-3}$ respectively at the equilibrium of $\mathrm{C}-\mathrm{Al}_{2} \mathrm{O}_{3}$ and $\mathrm{C}-\mathrm{CaO}$ systems. It is to be noted that these partial pressures can be the maximum estimates at the lower limit of $\mathrm{SiO}_{2}$ content in the slag.

The lower limit of the temperature, above which the contaminant content is high, shifted to higher values as $V_{\text {cg }}$ decreased. For example, it was about $1700 \mathrm{~K}$ at $V_{\text {cg }}=3.4 \times$ $10^{-6} \mathrm{Nm}^{3} \cdot \mathrm{s}^{-1}$ and $h=0.04 \mathrm{~m}$. Below the lower limit temperature, the content of metal impurities was very low according to the results of EPMA analysis.

The collected deposit was analyzed for $\mathrm{Si}, \mathrm{O}$ and $\mathrm{C}$ compositions by EPMA/WDX technique. To be able to measure the carbon concentration, the samples were coated with $\mathrm{Al}$ instead of $\mathrm{C}$ which is commonly used for sample coating. Figure 5 presents the temperature dependence of $\mathrm{Si}, \mathrm{O}$ and $\mathrm{C}$ concentrations in the deposit collected at locations of very low level of $\mathrm{Al}$ and $\mathrm{Ca}$ impurities except for two dots at $T>1600 \mathrm{~K}$. In the figure, each value was obtained by averaging the results of $4 \sim 5$ measurements. As seen from this figure, carbon concentration becomes significantly lower as the deposition temperature decreases. The silicon concentration,on the contrary, is slightly increased with the decrease in temperature. The deposit on the filter contains about $54 \mathrm{~mol} \%$ of $\mathrm{O}$ and $45 \mathrm{~mol} \%$ of Si while the carbon concentration is less than $1 \mathrm{~mol} \%$. In this case, molar ratio of $\mathrm{O}$ to $\mathrm{Si}$ is 1.2 indicating that the deposit composition is close to silicon monoxide.

\subsection{Deposit morphology}

Results of SEM analysis showed that the deposit presents nanostructured material of three types with different morphologies: nanoparticles, nanoparticle chain and nanowires depending on location where the deposit was collected. The deposit contained also comparatively large spherical particles 


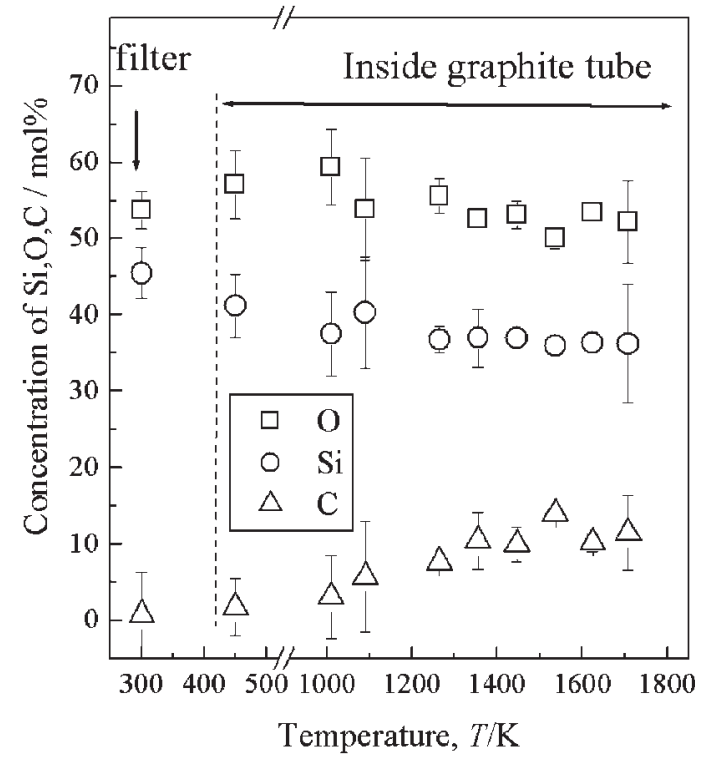

Fig. 5 Variation in $\mathrm{Si}, \mathrm{O}$ and $\mathrm{C}$ concentration with temperature.

with size ranging from 100 to $1000 \mathrm{~nm}$. However, they were not typical under the present experimental conditions and were observed in a small quantity only at larger gas flow rates.

Figure 6 presents SEM views of deposit grown on the inner surfaces of graphite tube under the same conditions as those for the data shown in Fig. 4: flow rate of carrier-gas, $V_{\text {cg }}=1.77 \times 10^{-5} \mathrm{Nm}^{3} \cdot \mathrm{s}^{-1}$, and tube position, $h=0.04 \mathrm{~m}$. The nanoparticles (Fig. 6(a)) were found only at lower temperatures of $T<920 \mathrm{~K}$. Their size was estimated as $40 \sim 60 \mathrm{~nm}$. The deposit on the filter also consisted of nanoparticles of the same size and morphology. Nanowires were dominated at higher temperatures. Figure 6(c) shows nanowires grown at $1490 \sim 1580 \mathrm{~K}$ where the largest amount of deposit was obtained. In the intermediate temperature range, the deposit consisted mainly of straight or slightly curved nanoparticle chains of variable length as exemplified in Fig. 6(b) for 1220 1320 K.

It was found that carrier gas flow rate, $V_{\mathrm{cg}}$, and tube position, $h$, are of prime importance in controlling the size and morphology of the nanostructures. At smaller $h$, $(0.04 \sim 0.045 \mathrm{~m})$, the size of nanoparticles increased with decreasing carrier gas flow rate, $V_{\mathrm{cg}}$. However, when $V_{\mathrm{cg}}$ became smaller than $3.4 \times 10^{-6} \mathrm{Nm}^{3} \cdot \mathrm{s}^{-1}$, no single particles were observed inside the reaction chamber because the particles formed chains. The similar result was obtained at larger $h,(>0.07 \mathrm{~m})$ even at larger $V_{\mathrm{cr}}$. When $h$ was larger than $0.145 \mathrm{~m}$, only nanowires of $30 \sim 50 \mathrm{~nm}$ in diameter were observed on the filter and surfaces inside the tubes.

There was a tendency for the nanowire length to increase with $h$. When the deposit was grown on the crucible walls, it consisted of long and smooth nanowires regardless of gas flow condition. An example of the wires are presented in Fig. 6(d). It is noteworthy that the deposit color was changed from brown through light yellow to light gray as the particles turned into the chains and nanowires.

\subsection{The process kinetics and mechanism}

As readily seen from the previous section, the most reactions involved occur with $\mathrm{CO}$ formation. Information on a)

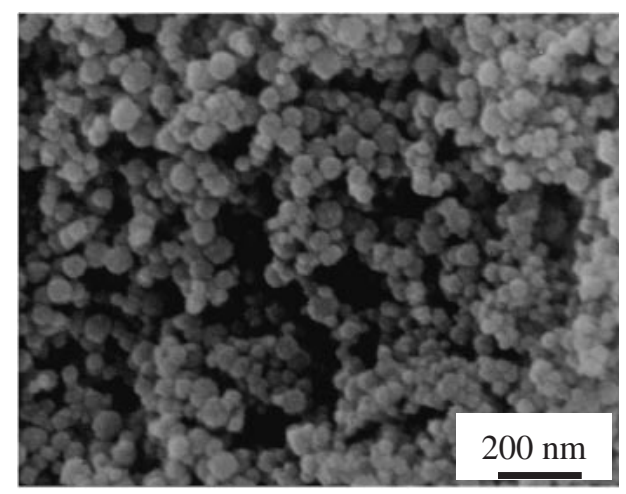

c)

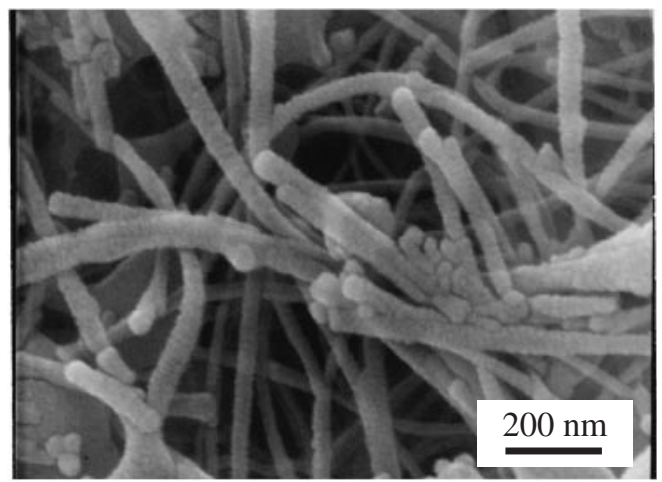

b)

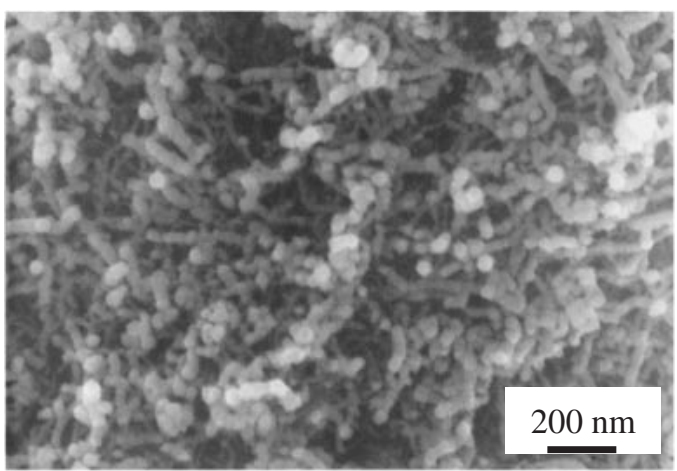

d)

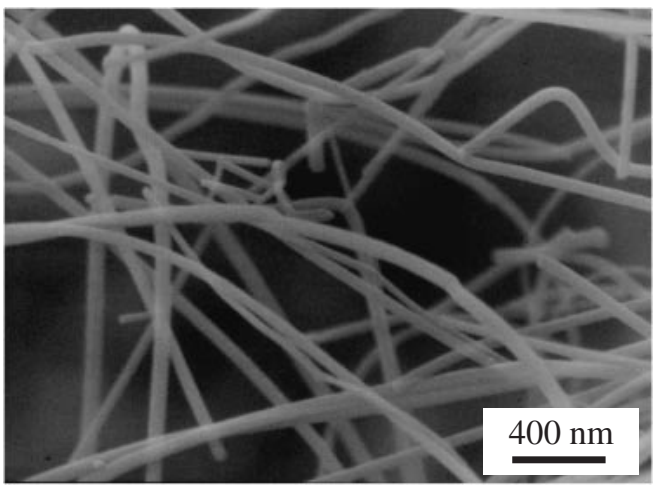

Fig. 6 SEM views of as-obtained deposit at different temperatures inside tube: a) $<920 \mathrm{~K}$, b) $1220 \sim 1320 \mathrm{~K} \mathrm{c}) 1490 \sim 1580 \mathrm{~K}$, and d) on crucible wall. 


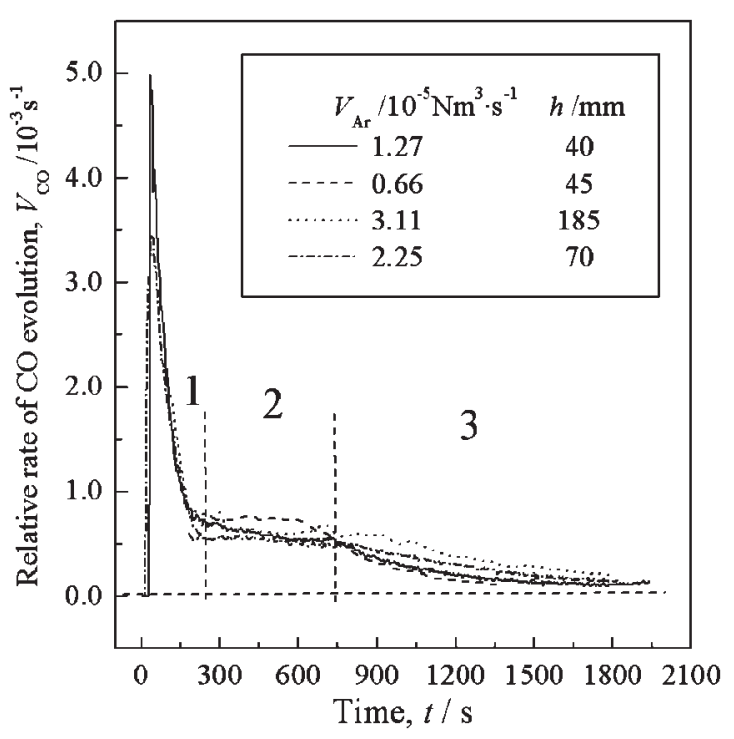

Fig. 7 Change in $\mathrm{CO}$ gas outflow rate with time.

$\mathrm{CO}$ evolution rate would be helpful for an understanding of kinetic aspects of the reaction system. The rate was determined from measurements of $\mathrm{CO}$ concentration in exhaust gas and gas volume as explained above. Figure 7 presents time variations in the rate of $\mathrm{CO}$ gas, $V_{\mathrm{CO}}$, leaving the crucible under various experimental conditions. Inasmuch as the $\mathrm{CO}$ outflow rate was inherently dependent on the total weight of pellets, $V_{\mathrm{CO}}$ was expressed in a dimensionless form as molar rate of $\mathrm{CO}$ divided by molar quantity of $\mathrm{SiO}_{2}$ in the initial oxide-carbon mixture. The time dependence of $V_{\mathrm{CO}}$ can be subdivided into three periods as indicated by corresponding figures and broken lines in Fig. 7. During the first period, the $\mathrm{CO}$ rate increased up to a maximum value, whereupon abruptly decreased for a time not longer than $5 \mathrm{~min}$. Then, the rate was kept constant with time during the second period. Duration of the second period was about $10 \mathrm{~min}$. The last third period was characterized by the further decrease in $V_{\mathrm{CO}}$. Such a variation in $\mathrm{CO}$ outflow rate suggests that conditions for reactions to proceed in the given system are changed with time.

To gain more detailed information about the reaction mechanism, a series of experiments was conducted under the same conditions except the reaction time. The conditions were $V_{\text {cg }}=1.27 \times 10^{-5} \sim 1.65 \times 10^{-5} \mathrm{Nm}^{3} \cdot \mathrm{s}^{-1}, h=0.04 \mathrm{~m}$ and $T=2073 \mathrm{~K}$. In two experiments, the reaction was stopped at 5 and $10 \mathrm{~min}$, respectively, with a rapid cooling down of the reaction system by the cut of the heater power supply. In the third experiment, the reaction time was $30 \mathrm{~min}$ that was the standard duration in the present study. After experiments, the crucibles were cut lengthwise to make sections containing residue in the crucible. Then, the residue was characterized by XRD and EPMA and, additionally, the residue constitution was examined with an optical microscope.

Figure 8 shows the XRD patterns of residual materials for all the three experiments. Two tendencies can be clearly seen in this figure. The first is the $\mathrm{SiC}$ formation beginning at the very early stage of the reduction process. This is confirmed by a few peaks of relatively low intensity of SiC observed

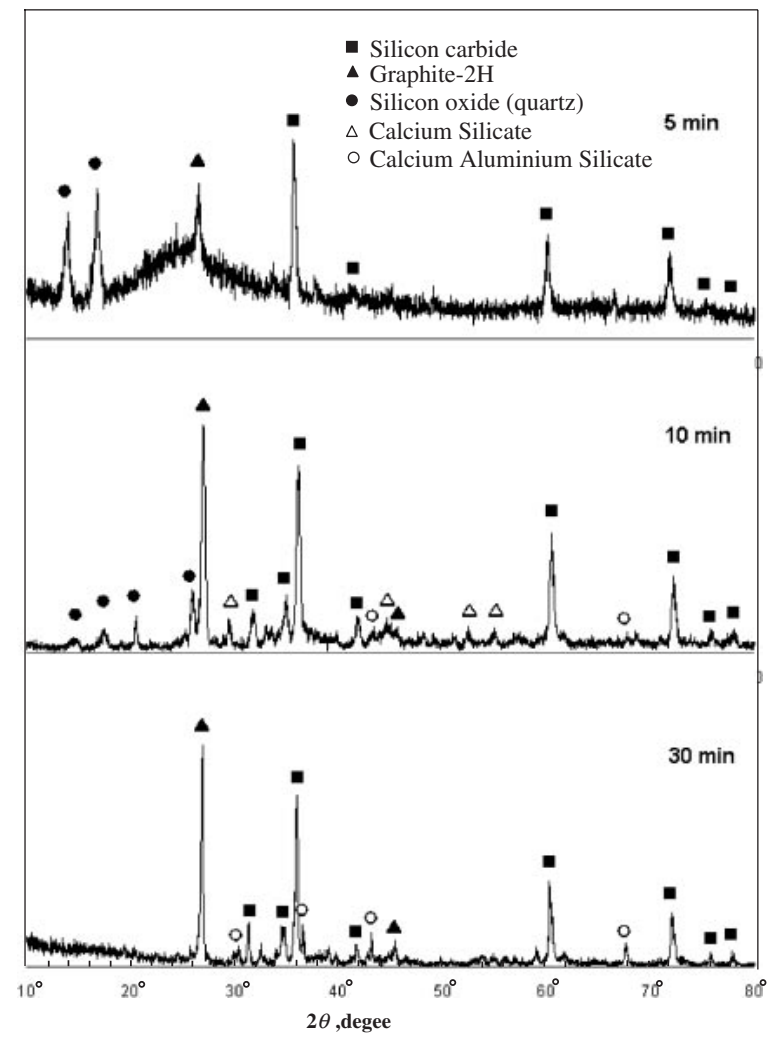

Fig. 8 XRD patterns of as-quenched residue in crucible.

already at the XRD for the experiment of $5 \mathrm{~min}$. The second tendency is a rapid decrease in amount of glassy phase in the slag melt with the reaction time. In the case of $5 \mathrm{~min}$, a broad background in the XRD pattern indicates the presence of amorphous glassy phase in significant amount. At $10 \mathrm{~min}$, on the other hand, according to the XRD pattern, the amount of the glassy phase is very small. It should be noted that we could not identify exactly some peaks in the XRD patterns which correspond supposedly to complex crystalline oxides of Al-Ca-Si system. Some possible compositions are indicated in the figure.

The similar tendencies can be observed in the digital microscope photographs of the cross-section of the asquenched residues as shown in Fig. 9. Each photograph presents typical images for 5, 10 and $30 \mathrm{~min}$, respectively. At $5 \mathrm{~min}$, the residue presents glassy layer observed as black background in Fig. 9 containing a lot of fine particles viewed as white dots in the figure. To verify the particle composition, the cross-section of the residue was selectively analyzed for the contents of $\mathrm{Si}, \mathrm{Al}$ and $\mathrm{Ca}$ by EDX. According to the analysis results, the particles of $3 \sim 5 \mu \mathrm{m}$ in size were enriched with $\mathrm{Si}$, and conversely were depleted with $\mathrm{Al}$ and $\mathrm{Ca}$ as compared to the composition of the glass matrix. Calculations showed that chemical composition of the particles was very close to that of SiC. Hence, the fine white particles in Fig. 9(a) are most likely to be $\mathrm{SiC}$ inclusions. At $10 \mathrm{~min}$, quantity of SiC inclusions increased significantly, and their shape became more acicular. At $30 \mathrm{~min}$, the residue was abundant of $\mathrm{SiC}$ which looks gray colored clusters consisting of very fine inclusions in Fig. 9(c). At this reaction time, EPMA results revealed that minor volume of glassy phase 

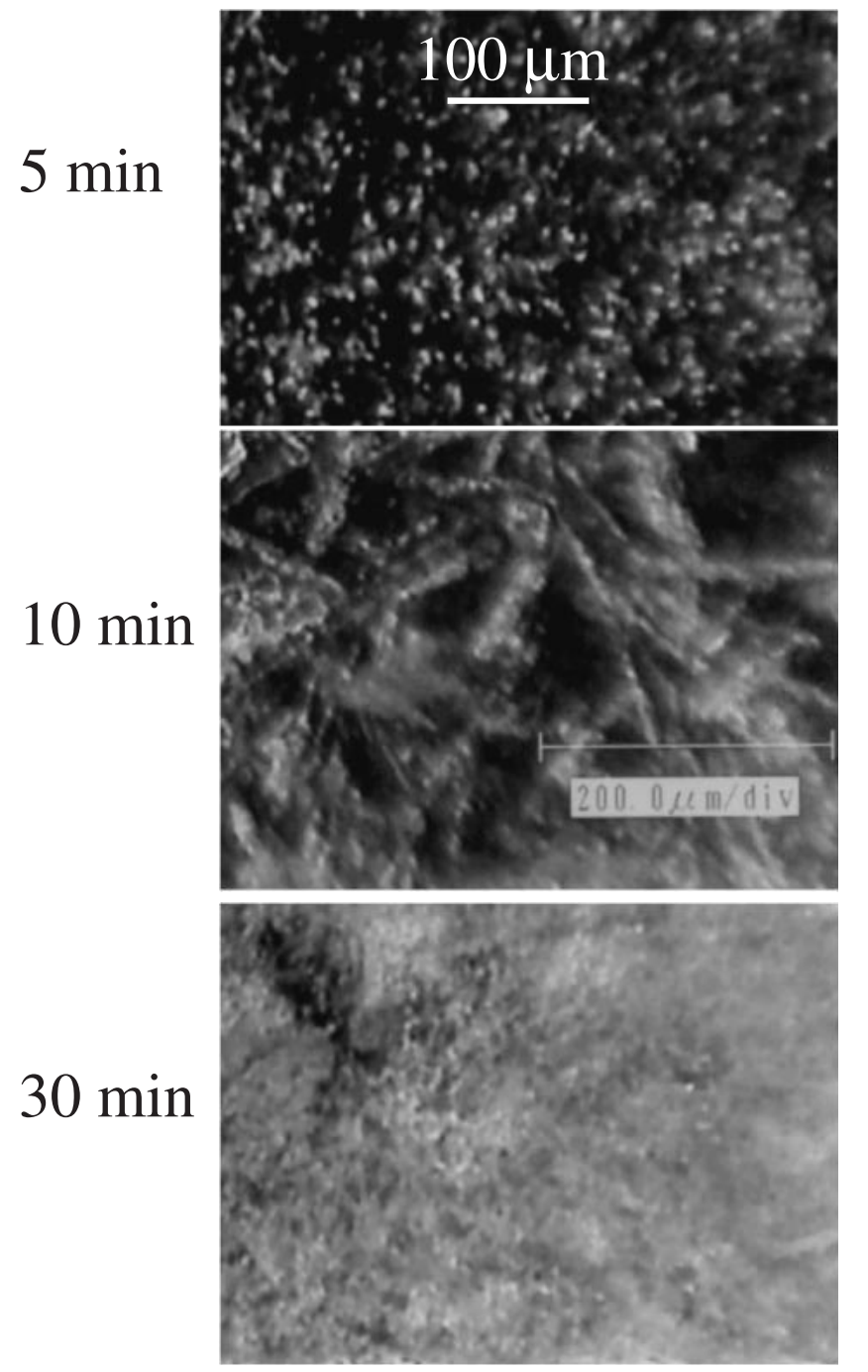

Fig. 9 Appearance of slag residue in crucible after experiment.

contain about 5 mass $\%$ of $\mathrm{SiO}_{2}, 31$ mass $\%$ of $\mathrm{CaO}$ and 64 mass $\%$ of $\mathrm{Al}_{2} \mathrm{O}_{3}$.

Thus, the three periods of the process in Fig. 7 can be explained as follows. At the very first stage, the vigorous emission of $\mathrm{CO}$ occurs because $\mathrm{SiO}_{2}$ of the melt reacts directly with carbon on the still-fresh surfaces of graphite particles or crucible according to reactions (1) and (2). This results in converting the carbon surfaces to $\mathrm{SiC}$. Since the $\mathrm{SiC}$ layer offers resistance to diffusion of $\mathrm{C}$ and $\mathrm{Si}$ at the solid side interface, mass transfer of the reaction reagents through the layer starts to play an increasingly important role in controlling the overall reaction rate. As a result, the $\mathrm{SiO}_{2}$ reduction rate and thus $\mathrm{CO}$ formation rate are decreased as the $\mathrm{SiC}$ layer thickness increases. This decrease can be observed in Fig. 7 during the last stage of the first period. In the second period, the very weak time dependence of $\mathrm{CO}$ emission rate can be explained from the assumption that the growing $\mathrm{SiC}$ layer can contain pores which could maintain a fast diffusion of gaseous species to the reaction zone. This assumption can be confirmed by the following experimental observations and by the data reported in the relevant literature. ${ }^{8)}$

Slag residue and parts of crucible bottom adjacent to the

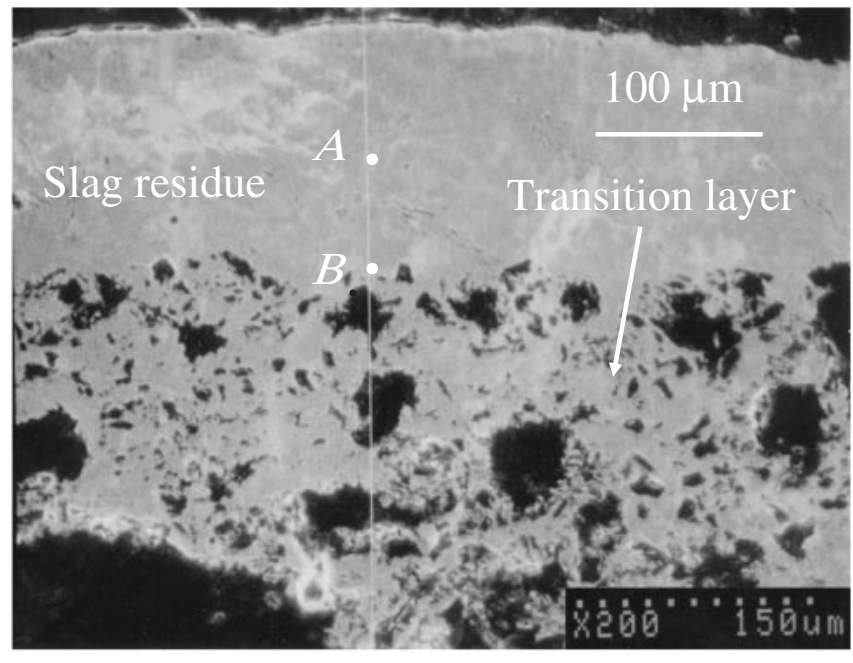

Fig. 10 A view of transition layer between slag and crucible bottom.

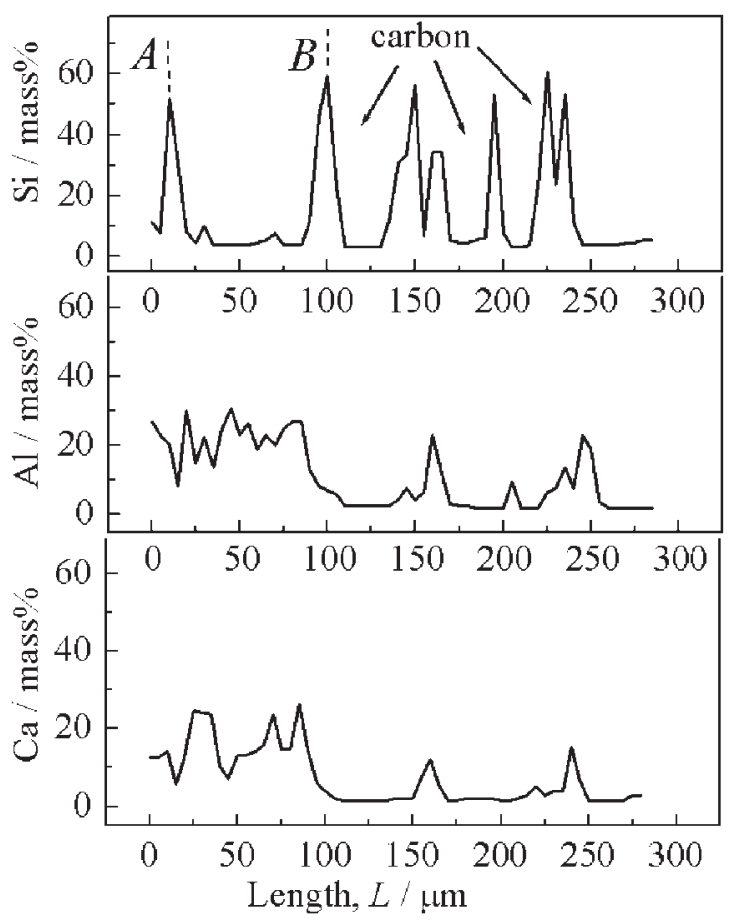

Fig. 11 EDS profiling of slag and transition layers.

slag were investigated by EPMA. The results indicated existence of a transition layer between the slag residue and graphite of the crucible. At the center of crucible bottom, the layer thickness was $250 \sim 300 \mu \mathrm{m}$ at $5 \mathrm{~min}$ and $400 \sim 500 \mu \mathrm{m}$ at 10 min. Figure 10 shows a SEM view of a part of transition layer at $10 \mathrm{~min}$. To examine the in-depth distribution of the layer components, EDX profiling was performed for $\mathrm{Si}, \mathrm{Al}$ and $\mathrm{Ca}$. Figure 11 shows the profiling results. Slag residue region (A-B, Fig. 10), except a small area around point $\mathrm{A}$ is rich in $\mathrm{Al}$ and $\mathrm{Ca}$ and poor in $\mathrm{Si}$. On the other hand, the transition layer (region below B) is enriched with $\mathrm{Si}$ and carbon while concentrations of $\mathrm{Al}$ and $\mathrm{Ca}$ are much less. Here, higher concentrations of $\mathrm{Si}$ at the peaks suggest that it exists in the transition layer in the form of $\mathrm{SiC}$. This was confirmed also by additional EPMA/WDX measurements. 
The zones of the transition layer between the $\mathrm{SiC}$ peaks were found to be composed of carbon. In the Fig. 10, these zones look as black spots.

These observations imply that the reaction of SiC formation can proceed in the bulk of crucible bottom. This is supported by the data on reaction mechanism between $\mathrm{SiO}_{2}$ and graphite available in the literature. Paccaud and Derre ${ }^{8)}$ reported that, on heating a $\mathrm{SiO}_{2}-\mathrm{SiC}$ powder mixture with a carbon piece up to temperatures of $1673 \sim 1973 \mathrm{~K}$, formation of $\mathrm{SiC}$ on the carbon surface occurred due to gaseous diffusion of $\mathrm{SiO}$ onto fresh carbon surface through connected pores in the carbon piece. These pores are grown intrinsically since the $\mathrm{SiC}$ formation causes stress due to a difference in density between $\mathrm{C}$ and $\mathrm{SiC}$. The stress originates the generation and propagation of cracks inside the bulk of graphite. Then, a newly generated fresh surface of graphite is exposed to gaseous $\mathrm{SiO}$ resulting in formation of new portions of SiC. Therefore, keeping the rate of $\mathrm{CO}$ emission during the second period of the reduction process (Fig. 7) can be explained by a recurring renewal of surface for the reaction between $\mathrm{SiO}$ and $\mathrm{C}$ inside the carbon particles or graphite crucible parts. The further decrease in reduction rate during the third period at Fig. 7 is probably associated with a significant diminish of $\mathrm{SiO}_{2}$ amount in the system due to its consumption for the reactions.

\section{Conclusions}

The experimental verification of the smelting reduction process showed that nanosized silicon based materials can be synthesized by carbothermic reduction of silica containing starting materials containing alumina and calcia. In the present study, the chemical composition of the starting materials was matched to the basic composition of silica-rich coal ash which is considered as a potentially usable source of $\mathrm{Si}$. The main point of the process is melting the starting materials at $2073 \mathrm{~K}$, reducing $\mathrm{SiO}_{2}$ to $\mathrm{SiO}$ vapor and carrying the vapor with $\mathrm{Ar}-\mathrm{CO}$ carrier gas mixture through a graphite tube from the highest temperature zone (above melt surface) to cooler parts of experimental reactor (filter and inner surfaces of the tube) where the vapor is condensed as $\mathrm{SiO}_{x}$ followed by further chemical transformations.

It is found that the degree of $\mathrm{SiO}_{2}$ reduction from slag, purity and morphology of the as-obtained nanoparticulates were governed by temperature and carrier-gas flow conditions. Higher flow rates of carrier gas and shorter distances between the graphite tube lower tip and melt surface were favor for $\mathrm{SiO}_{2}$ reduction. In these conditions, about 20 mass\% of $\mathrm{Si}$, contained initially in the molten slag as $\mathrm{SiO}_{2}$, was converted into $\mathrm{Si}$ in the form of nanostructured $\mathrm{Si}$ based material which was deposited on the inner tube surfaces and filter. Elemental analysis showed that the material consists of $\mathrm{Si}, \mathrm{O}$ and $\mathrm{C}$ with atomic fractions varying with the deposition temperature. As the temperature became lower, concentration of $\mathrm{C}$ is decreased while that of $\mathrm{Si}$ is slightly increased.

Depending on temperature, the material presented chiefly rounded nanoparticles $(<920 \mathrm{~K})$, nanoparticle chains (920 $1320 \mathrm{~K})$ or nanowires $(1320 \sim 1570 \mathrm{~K})$. The nanoparticulate diameter was ranged from 20 to $100 \mathrm{~nm}$ with a trend toward a decrease as the gas flow rate increases. The nanoparticles and nanochains were free of metal impurities while the purity of nanowires was dependent on deposition temperature. If the temperature exceeded a threshold value, the deposit was highly contaminated with metal impurities such as $\mathrm{Al}, \mathrm{Ca}, \mathrm{K}$ and Fe. Under the present experimental conditions, the threshold value was ranged from 1600 to $1700 \mathrm{~K}$ depending on carrier gas flow rate. At lower carrier-gas flow rate and/or larger tube distance, nanoparticles were transformed into the nanoparticle chains, which, in turn, were changed into the nanowires on further decreasing the carrier-gas flow rate and/or increasing the tube distance. However, the decrease in carrier-gas flow rate and/or increase in tube distance leaded to a significant drop in the degree of $\mathrm{SiO}_{2}$ reduction.

It is shown that the limitations in the efficiency of $\mathrm{SiO}_{2}$ reduction are associated with a strong tendency of $\mathrm{C}$ to react with $\mathrm{SiO}_{2}$ or $\mathrm{SiO}$ producing $\mathrm{SiC}$ in the present reaction system. About $50 \%$ of $\mathrm{Si}$ was converted into $\mathrm{SiC}$ under the given experimental conditions. Nevertheless, it is believed that the smelting reduction process is a promising candidate for potential application in the production of Si-based nanostructured materials due to its good controllability, high productivity and possibility for processing cheap and plentiful silica-containing materials.

\section{Acknowledgments}

The authors gratefully acknowledge the financial support for this work from Kobe Steel Ltd.

\section{REFERENCES}

1) Y. F. Zhang, Y. H. Tang, C. Lam, N. Wang, C. S. Lee, I. Bello and S. T. Lee: J. Crys. Growth 212 (2000) 115-118.

2) H. Hofmeister, P. Ködderitzsch and J. Dutta: J. Non-Cryst. Solids 232234 (1998) 182-187.

3) Y. Q. Zhu, W. K. Hsu, N. Grobert, M. Terrones, H. Terrones, H. W. Kroto, D. R. M. Walton and B. Q. Wei: Chem. Phys. Lett., 322 (2000) 312-320.

4) J. L. Gole, J. D. Stout, W. L. Rauch and Z. L. Wang: Appl. Phys. Lett., 76 (2000) 2346-2348.

5) Y. F. Zhang, Y. H. Tang, N. Wang, C. S. Lee, I. Bello and S. T. Lee: J. Crys. Growth 197 (1999) 136-140.

6) M. Tada and M. Hirasawa: High Temperature Materials and Processes 19 (2000) 281-297.

7) D. H. Filsinger and D. B. Bourrie: J. Am. Ceram. Soc. 73 (1990) 17261732.

8) O. Paccaud and A. Derre: Chem. Vapor Deposition 6 (2000) 41-50. 